\title{
SOME APPLICATIONS OF THE EULERIAN FUNGTIONS OF A FINITE GROUP
}

\author{
G. E. WALL
}

(received 27 July 1960)

\section{Introduction}

This paper is chiefly concerned with inequalities for the numbers of subgroups of a finite $p$-group. The following are typical results. Let $G$ be a $p$-group of order $p^{a}$, and let $n_{G}\left(p^{k}\right)$ denote the number of subgroups of $G$ of order $p^{k}$.

(1.1) If $A$ is the elementary abelian group of the same order as $G$, then $n_{G}\left(p^{k}\right) \leqq n_{A}\left(p^{k}\right)(k=1,2, \cdots)$. If equality holds for a value of $k$ such that $1<k<a$, then $G \cong A$.

(1.2) If $G$ is a regular p-group, and if $B$ is the abelian $p$-group with the same basis invariants as $G$, then $n_{G}\left(p^{k}\right) \leqq n_{B}\left(p^{k}\right)(k=1,2, \cdots)$. If $G$ has the same total number of subgroups as $B$, then $G$ is lattice-isomorphic to $B$.

We mention in passing that (at any rate for $p=2,3$ ) two $p$-groups may have the same number of subgroups of each order without being lattice-isomorphic. See $\S 7$, figs. 1 and 2.

Results like (1.1) and (1.2) are relevant to the problem of the "lattice embeddings" of p-groups: a lattice embedding of a group $G$ in a group $H$ is a meet isomorphism of the lattice of subgroups of $G$ into that of $H$. Whenever a $p$-group $G$ can be lattice-embedded in a $p$-group $H$ of the same order, the series of inequalities

$$
n_{G}\left(p^{k}\right) \leqq n_{H}\left(p^{k}\right) \quad(k=1,2, \cdots)
$$

is a necessary consequence; for such a lattice embedding maps each subgroup of $G$ onto a subgroup of $H$ of the same order. Lattice embeddings of $p$-groups have been recently studied by $D$. W. Barnes $([1])$.

(1.1) immediately suggests the possibility that every $p$-group $G$ can be lattice-embedded in the elementary abelian group of the same order; but Barnes has shown that this is false, even when $G$ is abelian. However, the problem for groups of exponent $p$, and the corresponding problem suggested 
by (1.2), remain open. With regard to the latter, Dr. Graham Higman has pointed out that the answer is affirmative when every subgroup of $G$ which can be generated by 3 elements has class less than $p$; this is an immediate consequence of a theorem of M. Lazard ([7]).

Our method is enumerative, and relies on the properties of the Eulerian functions of a group (cf. P. Hall, [4]). Let $G$ be any finite group. The $n$-th Eulerian function $\phi_{n}(G)$ of $G$ is defined to be the total number of sequences of $n$ elements of $G$ which generate $G$. By counting the number of sequences of $n$ elements of $G$ in two ways, we get the analogue of the Eulerian summation formula of arithmetic:

$$
\sum_{\boldsymbol{H} \leqq G} \phi_{n}(H)=|G|^{n}
$$

where summation is over the subgroups $H$ of $G$. The value of $\phi_{n}(G)$ is given explicitly by the inversion formula

$$
\sum_{H \leqq G} \mu_{G}(H)|H|^{n}=\phi_{n}(G)
$$

where $\mu_{G}$ is the Möbius function of $G$ (Hall, l.c.; L. Weisner, [9]). For technical reasons, we shall deal with the Eulerian polynomial $\phi(G)$ of $G$ rather than the series of Eulerian functions $\phi_{n}(G)(n=1,2, \cdots) . \phi(G)$ is a polynomial, in variables $x, y, \cdots$ corresponding to the distinct prime divisors $p, q, \cdots$ of $|G|$, which reduces to $\phi_{n}(G)$ for the particular values $x=p^{n}$, $y=q^{n}, \cdots$.

The inequality (1.1) is a fairly easy consequence of (1.3) in the case of a $p$-group (\$ 4). (1.2) follows similarly from a more general formula in which $\phi_{n}(G)$ is replaced by another function of Eulerian type $(\S 7)$. One by-product of our formulae is the (known) enumeration of the subgroups of given isomorphism type in an abelian group.

The above results for $p$-groups give some information about groups of composite order, for it is quite easy to get upper bounds (though rather crude ones) for the numbers of subgroups of a group in terms of the numbers of subgroups of its Sylow subgroups. E.g. if $|G|=p^{a} q^{b} \cdots$, and if $P, Q, \cdots$ are Sylow subgroups corresponding to the $t$ distinct prime divisors $p, q, \cdots$, then

$$
n_{G}\left(p^{\alpha} q^{\beta} \cdots\right) \leqq|G|^{t-1} \dot{n}_{P}\left(p^{\alpha}\right) n_{Q}\left(q^{\beta}\right) \cdot
$$

Again, if $G$ is soluble, $|G|^{t-1}$ can be replaced by $|G|$ in (1.5).

The problem of finding direct generalizations of results like (1.1), (1.2) to groups of composite order seems to be more difficult. First, our method succeeds for $p$-groups largely because of the simplicity of their Eulerian polynomials - the complexity of $\phi(G)$ depends on the complexity of the Frattini factor group $G / \Phi(G)$, which, for a p-group, is elementary abelian. Second, it is not easy to see which groups are to play the part of the "universal" groups 
$A, B$ in (1.1), (1.2). E.g. the simple group of order 168 has more subgroups of order 24 than any soluble group of order 168, though less of order 56 . Such considerations suggest that it may be more fruitful to restrict attention to composition subgroups.

Recently, W. Gaschütz ([3]) has proved an interesting theorem about the Eulerian polynomial of a soluble group $G$, viz. that

$$
\phi(G)=\rho(x) \sigma(y) \cdots,
$$

where the polynomials $\rho, \sigma, \cdots$ depend on the chief factors of $G$. Gaschütz's method shows, in fact, that if $G$ is $p$-soluble, then

$$
\phi(G)=\rho(x) \sigma(y, z, \cdots),
$$

where $\rho$ is determined just as in the soluble case. It would be very interesting to know whether every group with Eulerian polynomial of the form (1.6) is necessarily soluble. The concluding $\S 8$ contains several partial results on this problem. First, a group $G$ has a normal subgroup of prime index $p$ if, and only if, $(x-1)$ is a divisor of $\phi(G)$. It is easily deduced that if $\phi(G)$ has the form (1.6) then $G$ is not perfect, i.e. $G^{\prime}<G$. Second, if $\phi(G)$ has the form (1.6) and $\mu_{G}(1) \neq 0$, then $G$ is soluble. We remark that, for soluble groups $G, \mu_{G}(1) \neq 0$ if, and only if, the Frattini subgroup of every homomorphic image of $G$ is the identity (this follows from Gaschütz's formula for $\phi(G)$ ). On the other hand, $\mu_{G}(1)=-60$ when $G$ is the icosahedral group and $\mu_{G}(1)=0$ when $G$ is the simple group of order 168 .

We also prove in $\S 8$, using Gaschütz's methods, that the number of maximal subgroups of a soluble group is less than the group order. The same result for a general finite group would have important consequences. E.g. it would follow that every group $G$ which cannot be generated by 2 elements has a proper subgroup $H$ such that $|G: H|<|H|$.

\section{Sum Functions*}

Let $G$ be a finite group and $A$ an additively written abelian group. A subgroup function on $G$ to $A$ is a mapping of the lattice of subgroups of $G$ into $A$. In the sequel, $A$ is either the group of ordinary integers or the underlying group of a polynomial domain over the integers. The notations $H \leqq G, H<G, H \triangleleft G$ mean respectively that $H$ is a subgroup of $G$, a subgroup of $G$ distinct from $G$, a normal subgroup of $G$.

If the subgroup functions $g, h$ satisfy

$$
g(H)=\sum_{K \leq H} h(K)
$$

for all $H \leqq G$,

we call $g$ the sum function of $h$ and write

* For further details on the (known) results of this section see Hall [4], Gaschütz [3]. 


$$
g=\sum h \text {. }
$$

For given $g$, the system of equations (2.1) determines $h$ uniquely; for, if $h(K)$ is known for all $K<H$, then $h(H)$ is given by

$$
h(H)=g(H)-\sum_{\boldsymbol{K}<\boldsymbol{B}} h(K) .
$$

We call $h$ the summand function of $G$ and write

$$
h=\Sigma^{-1} g \text {. }
$$

The equations

$$
\mu_{G}(G)=1, \quad \sum_{K \geqq H} \mu_{G}(K)=0 \quad \text { whenever } H<G,
$$

define the Möbius function $\mu_{G}$ of $G ; \mu_{G}$ is a subgroup function on $G$ to the group of ordinary integers. It is easily verified that

$$
h(G)=\sum_{H \leqq G} \mu_{G}(H) g(H)
$$

by showing that $g$ is the sum function of the right hand side.

An explicit formula for $\mu_{G}(H)$ can be got as follows. Let $M_{1}, \cdots, M_{r}$ be the maximal subgroups of $G$ (not including $G$ itself). If $S=\left[i_{1}, \cdots, i_{8}\right]$ is a subset of $I=[1,2, \cdots, r]$, we write

$$
\begin{aligned}
(-1)^{S} & =(-1)^{s}, \\
M_{S} & =M_{i_{1}} \wedge M_{i_{1}} \wedge \cdots \wedge M_{i_{j}} ;
\end{aligned}
$$

in particular, $M_{\phi}=G$, and $M_{I}$ is the Frattini subgroup $\Phi(G)$ of $G$. Let $S_{H}$ denote the set of indices $i$ such that $H \leqq M_{i}$. Then

and so

$$
\begin{aligned}
& \sum_{M_{S} \geqq H}(-1)^{S}=\sum_{S \leqq S_{H}}(-1)^{S} \\
& \left.=\begin{array}{ll}
1 & (H=G) \\
0 & (H<G)
\end{array}\right\} \text {. }
\end{aligned}
$$

$$
\mu_{G}(H)=\sum_{M_{S}-H}(-1)^{S} .
$$

It follows that $\mu_{G}(H)=0$ unless $H$ is an intersection of maximal subgroups of $G$; in particular, $\mu_{G}(H)=0$ unless $\Phi(G) \leqq H$. In view of (2.6), we may write (2.5) as

$$
h(G)=\sum_{S \leq I}(-1)^{S} g\left(M_{S}\right) .
$$

We remark that if $N \triangleleft G, N \leqq H \leqq G$, then

$$
\mu_{G / N}(H / N)=\mu_{G}(H) \text {. }
$$

This is clear from the defining equations (2.4). 


\section{The Eulerian Polynomial}

Let $G$ be a finite group of order $p^{a} q^{b} \cdots$, where $p, q, \cdots$ are distinct primes. Choose variables $x, y, \cdots$ corresponding to $p, q, \cdots$. (When it is necessary to indicate the precise correspondence between primes and variables, we shall write $x_{p}, x_{q}, \cdots$ instead of $\left.x, y, \cdots\right)$ We define the order polynomial $f(G)$ to be

$$
f(G)=f(G ; x, y, \cdot)=x^{a} y^{b} \cdot \cdot
$$

and the Eulerian polynomial $\phi(G)$ to be the summand function of $f(G)$ :

$$
\phi(G)=\sum_{H \leqq G} \mu_{G}(H) f(H) .
$$

Since $\mu_{G}(H)=0$ unless $H$ contains the Frattini subgroup $\Phi(G)$ of $G$, we have

$$
\phi(G)=\phi(G / \Phi(G)) f(\Phi(G)) .
$$

Thus, e.g., in order to calculate the Eulerian polynomial of a $p$-group it is sufficient to know the Eulerian polynomial of an elementary abelian group.

Since $\phi_{n}(G)$ is the summand function of

we have

$$
|G|^{n}=f\left(G ; p^{n}, q^{n}, \cdot \cdot\right)
$$

$$
\phi_{n}(G)=\phi\left(G ; p^{n}, q^{n}, \cdots\right) \quad(n=0,1, \cdots) .
$$

In particular,

Since

$$
\phi(G ; 1,1, \cdot)=0
$$

$$
\begin{aligned}
& \left.f(G ; 0,0, \cdots)=\begin{array}{ll}
1 & (G=1) \\
0 & (G>1)
\end{array}\right\}, \\
& \phi(H ; 0,0, \cdots)=\mu_{G}^{*}(H),
\end{aligned}
$$

where $\mu_{G}^{*}$ is the "dual" Möbius function of $G$ (Hall, [4]). By (3.2),

$$
\phi(G ; 0,0, \cdots)=\mu_{G}^{*}(G)=\mu_{G}(1) .
$$

THEOREM $1 . \dagger$ If $N \triangleleft G$,

$$
f(N) \phi(G / N)=\sum_{\boldsymbol{H}=G} \phi(H) .
$$

Proof.

$$
\begin{aligned}
f(N) \phi(G / N) & =\sum_{K / N \leqq G / N} f(N) \mu_{G / N}(K / N) f(K / N) \\
& =\sum_{K \geq N} \mu_{G}(K) f(K) \\
& =\sum_{K \geq N} \mu_{G}(K) \sum_{H \leq K} \phi(H) \\
& =\sum_{H \leq G} \phi(H) \sum_{K \leq N H} \mu_{G}(K) \\
& =\sum_{N=G} \phi(H) .
\end{aligned}
$$

t This theorem is a slight variant of theorem 1 in Gaschütz [3]. 
E.g., let $G$ be the symmetric group $S_{4}, N$ its normal subgroup of order 4; then $x_{2}^{2} \phi\left(S_{3}\right)=\phi\left(S_{4}\right)+4 \phi\left(S_{3}\right)$, so that $\phi\left(S_{4}\right)=\left(x_{2}^{2}-4\right) \phi\left(S_{3}\right)=$ $\left(x_{2}-1\right)\left(x_{2}^{2}-4\right)\left(x_{3}-3\right)$. Thus, there are $\left(2^{2}-1\right)\left(2^{4}-4\right)\left(3^{2}-3\right)=216$ ordered pairs of generators of $S_{4},\left(2^{3}-1\right)\left(2^{6}-4\right)\left(3^{3}-3\right)=10080$ ordered triples of generators, etc.

Corollary.

$$
\phi(G / N) \mid \phi(G) .
$$

Proof. We may assume, by induction on the group order, that each term on the right of (3.6) except $\phi(G)$ is divisible by $\phi(G / N)$, for

$$
H / H \wedge N \cong G / N \quad \text { if } H N=G \text {. }
$$

Hence, by (3.6),

$$
\phi(G / N) \mid \phi(G)
$$

\section{Numbers of Subgroups of a p-Group}

In the present section, $G$ is a $p$-group such that

$$
|G|=p^{m}, \quad|G: \Phi(G)|=p^{d} .
$$

The significance of the index $d$ is that $G$ can be generated by $d$, but no fewer, elemen s. We shall therefore say that $G$ is a $d$-generator group.

Let $\alpha_{k}$ denote the elementary abelian group of order $p^{k}$. If $A_{1}$ is any subgroup of $A_{k}$ of order $p$, there are $p^{k-1} A_{k-1}$ 's such that $A_{1} A_{k-1}=A_{k}$. Therefore, by theorem 1 ,

i.e.

$$
x \phi\left(A_{k-1}\right)=\phi\left(A_{k}\right)+p^{k-1} \phi\left(A_{k-1}\right),
$$

Hence

$$
\phi\left(A_{k}\right)=\left(x-p^{k-1}\right) \phi\left(A_{k-1}\right) .
$$

where

$$
\phi\left(A_{k}\right)=X_{k}(x),
$$

$$
X_{k}(x)=\prod_{\lambda=0}^{k-1}\left(x-p^{\lambda}\right) .
$$

Now, $G / \Phi(G) \cong A_{d}$. Therefore, by (3.3),

$$
\phi(G)=x^{m-d} X_{a}(x) .
$$

In view of (4.1), the Euler summation formula for $G$ can be written

$$
x^{m}=\sum_{r, s} N_{r, s}(G) x^{s-r} X_{r}(x),
$$

where $N_{r, s}(G)$ is the number of $r$-generator subgroups of $G$ of order $p^{s}$. The identity obtained by putting $x=0$ in (4.2) is perhaps of some interest:

$$
\sum_{k=0}^{m}(-1)^{k} p^{\frac{1}{2} k(k-1)} a_{k}(G)=0,
$$


where $a_{k}(G)$ is the number of elementary abelian subgroups of $G$ of order $p^{k}$. Taking $G=A_{m}$ in (4.2), we get

$$
x^{m}=\sum_{k=0}^{m} \omega(m, k) X_{k}(x),
$$

where

$$
\omega(m, k)=\omega(m, m-k)=\frac{\left(p^{m}-1\right)\left(p^{m-1}-1\right) \cdots\left(p^{m-k+1}-1\right)}{\left(p^{k}-1\right)\left(p^{k-1}-1\right) \cdots(p-1)}
$$

is the number of subgroups of $A_{m}$ of order $p^{k}$. By (2.8) and (3.5), the Möbius function $\mu=\mu_{A_{m}}$ of $A_{m}$ is given by

$$
\mu\left(A_{m-k}\right)=(-1)^{k} p^{\frac{1}{2} k(k-1)} .
$$

Hence the inversion formula corresponding to $(4.3)$ is

THEOREM 2.

$$
X_{m}(x)=\sum_{k=0}^{m}(-1)^{k} \omega(m, k) p^{\frac{1}{2 k(k-1)}} x^{m-k}
$$

$$
\omega(m, k)=\sum_{r \leqq k \leqq s} \omega(s-r, k-r) p^{r(s-k)} N_{r, s}(G) \quad(0 \leqq k \leqq m) .
$$

Proof. Replacing $x$ by $x p^{-t}$ in (4.3) and then simplifying, we get,

$$
x^{m} X_{t}(x)=\sum_{k=0}^{m} \omega(m, k) p^{t(m-k)} X_{t+k}(x) .
$$

Using $(4.5)^{\prime}$, we can write $(4.2)$ as

$$
x^{m}=\sum_{r, s} N_{r, s}(G) \sum_{k=r}^{s} \omega(s-r, k-r) p^{r(s-k)} X_{k}(x) .
$$

Comparison of the coefficients of $X_{k}(x)$ in (4.3) and (4.6) now gives the theorem.

Let $n_{k}(G)$ denote * the number of subgroups of $G$ of order $p^{k}, N_{k}(G)$ the number of subgroups of $G$ which can be generated by $k$ (or fewer) elements and have order $\geqq p^{k}$. Since

we have

$$
\begin{aligned}
n_{k}(G) & =\sum_{r \leqq k} N_{r, k}(G), \\
N_{k}(G) & =\sum_{r \leqq k \leqq s} N_{r, s}(G),
\end{aligned}
$$

COROLlary 1.

$$
\begin{gathered}
N_{k}(G) \leqq N_{k}\left(A_{m}\right)=\omega(m, k) . \\
n_{k}(G) \leqq n_{k}\left(A_{m}\right)=\omega(m, k) .
\end{gathered}
$$

Corollary 2.

* The notation $n_{k}(G)$ is more convenient for our present purposes than the systematic notation $n_{G}\left(p^{k}\right)$ used in $\S 1$. 
Since $\omega(m, 1)=\left(p^{m}-1\right) /(p-1)$, it is clear that $N_{1}(G)=N_{1}\left(A_{m}\right)$ if, and only if, $G$ has exponent $p$. On the other hand, if $N_{k}(G)=N_{k}\left(A_{m}\right)$ and $1<k<m$ then $G \cong A_{m}$. For, by (4.5), $N_{r, s}(G)=0$ whenever $0<r \leqq k$ $<s$. It follows that

(a) every $r$-generator subgroup of $G(r \leqq k)$ has order $\leqq p^{k}$;

(b) every subgroup of $G$ of order $p^{k+1}$ is elementary abelian.

Let $x, y \in G$. By (a), and because $k \geqq 2,|\{x, y\}| \leqq p^{k}$. Therefore, by (b), $\{x, y\}$ is contained in an elementary abelian subgroup of $G$. Hence $x^{p}=y^{p}=x y x^{-1} y^{-1}=1$, and so $G$ is elementary abelian.

Theorem 2 throws some light on the well known enumeration theorems of $p$-group theory. E.g., let $C_{r}(G)$ denote the number of cyclic subgroups of $G$ of order $p^{r}$; taking congruences $\left(\bmod p^{2}\right)$ in $(4.5)$, we get, for $0<k \leqq m$,

$$
n_{k}(G)+C_{k+1}(G) p \equiv \omega(m, k)
$$

and so, for $0<k<m$,

$$
n_{k}(G)+C_{k+1}(G) p \equiv 1+p
$$

(4.8) is to be compared with the theorems of Kulakoff and Miller that, when $G$ is non-cyclic, $p>2$ and $0<k<m$,

$$
\begin{aligned}
n_{k}(G) & \equiv 1+p\left(\bmod p^{2}\right), \\
C_{k+1}(G) & \equiv 0(\bmod p) .
\end{aligned}
$$

Again, if $0 \leqq k \leqq d$ and if $G$ is not elementary abelian, (4.5) yields the congruence

$$
n_{m-k}(G) \equiv \omega(m, k)-N_{d-k+1, m-k+1}(G) p^{d-k+1} \quad\left(\bmod p^{d-k+2}\right) ;
$$

$N_{d-k+1, m-k+1}(G)$ being the number of subgroups $H$ of $G$ of order $p^{m-k+1}$ such that $\Phi(H)=\Phi(G)$. This is a slight refinement of P. Hall's congruence

(Cf. Zassenhaus [11]).

$$
n_{m-k}(G) \equiv \omega(d, k)
$$

$\left(\bmod p^{d-k+1}\right)$.

\section{The Eulerian Polynomial of a Chain}

In the present section, $G$ is once more an arbitrary finite group. The general Eulerian polynomials considered below are, so to speak, "polarized" forms of the basic Eulerian polynomial $\phi(G)$.

Let

$$
\mathscr{G}: 1=G_{0} \leqq G_{1} \leqq \cdots \leqq G_{r}=G
$$

be a chain of subgroups, of formal length $r$, joining the identity to $G$. If $H \leqq G$, we call

- (4.7) reduces to $1+p \equiv \omega(m, k)\left(\bmod p^{2}\right)$ when $G$ is the cyclic group of order $p^{m}$. 


$$
\mathscr{H}: 1=H_{0} \leqq \cdots \leqq H_{r}=H,
$$$$
\left(H_{i}=G_{i} \wedge H\right)
$$

the subchain of $\mathscr{G}$ corresponding to $H$. If $H \triangleleft G$, we call

$$
\mathscr{G} \mid \mathscr{H}: 1=(G / H)_{0} \leqq \cdots \leqq(G / H)_{r}=\dot{G} / H \quad\left((G / H)_{i}=G_{i} H / H\right)
$$

the factor chain of $\mathscr{G}$ corresponding to $G / H$. We write $\mathscr{H} \leqq \mathscr{G}$ when $H \leqq G$, $\mathscr{H} \triangleleft \mathscr{G}$ when $H \triangleleft G$. Corresponding subgroups and subchains are denoted by corresponding Italian and script capitals. The following easily proved facts justify this convention:

(a) if $H \leqq K \leqq G$, then $\mathscr{H} \leqq \mathscr{K}$;

(b) if $H \triangleleft G$ and $H \leqq K \leqq G$, then $\mathscr{K}|\mathscr{H} \leqq \mathscr{G}| \mathscr{H}$;

(c) if $H \triangleleft G, K \triangleleft G$ and $H \leqq K \leqq G$, then the natural homomorphism of $(G / H) /(K / H)$ onto $G / K$ maps $(\mathscr{G} / \mathscr{H}) /(\mathscr{K} / \mathscr{H})$ onto $\mathscr{G} / \mathscr{K}$.

The subchains corresponding to the subgroups $H K \cdots$ (where $H, K, \cdots$ are permutable), $H \wedge K \wedge \cdots,\{H, K, \cdots\}$ are denoted by $\mathscr{H} \mathscr{K} \cdots$, $\mathscr{H} \wedge \mathscr{K} \wedge \cdots,\{\mathscr{H}, \mathscr{K}, \cdots\}$ respectively.

We define now the order, and Eulerian, polynomials of the chain $\mathscr{G}$. For each "link" $G_{i-1} \leqq G_{i}$, we choose variables $x_{i}, y_{i}, \cdots$ corresponding to the distinct prime divisors $p, q, \cdots$ of $|G|$. Let

$$
\left|G_{i}: G_{i-1}\right|=p^{a_{i}} q^{b_{i}} \ldots \quad \quad(1 \leqq i \leqq r),
$$

and let $\xi_{i}$ stand collectively for the variables $x_{i}, y_{i}, \cdots$. Then we define

$$
\begin{gathered}
f(\mathscr{G})=f\left(\mathscr{G} ; \xi_{1}, \cdots, \xi_{r}\right)=\prod_{i=1}^{r}\left(x_{i}^{a_{i}} y_{i}^{b_{t}} \cdots\right), \\
\phi(\mathscr{G})=\Sigma^{-1} f(\mathscr{G}) .
\end{gathered}
$$

The domain of summation in (5.5) is the set of subchains of $\mathscr{G}$ or, what is essentially the same, the set of subgroups of $G$. Thus,

$$
\phi(\mathscr{G})=\sum_{H \leq G} \mu_{G}(H) f(\mathscr{H}) .
$$

Clearly, $\phi(\mathscr{G})$ reduces to $\phi(G)$ when $r=1$.

As before, $\phi(\mathscr{G})$ corresponds to a series of (generalized) Eulerian functions. Let $N=\left(N_{1}, \cdots, N_{r}\right)$ be a row of $r$ integers such that $N_{1} \geqq N_{2} \geqq \cdots \geqq$ $N_{r} \geqq 0$. Write

so that

$$
n_{i}=N_{i}-N_{i+1} \quad\left(1 \leqq i \leqq r ; N_{r+1}=0\right),
$$

$$
N_{i}=\sum_{j=i}^{r} n_{j}
$$$$
(1 \leqq i \leqq r)
$$

By an $N$-sequence for $\mathscr{G}$ we shall mean a sequence of $N_{1}$ elements of $G$ whose first $n_{1}$ members belong to $G_{1}$, next $n_{2}$ to $G_{2}$, etc. By a generating $N$-sequence for $\mathscr{G}$ we mean an $\boldsymbol{N}$-sequence for $\mathscr{G}$ whose members generate $G$. The $\boldsymbol{N}$-th Eulerian 
function $\phi_{N}(\mathscr{G})$ of $\mathscr{G}$ is defined as the total number of generating $N$-sequences for $\mathscr{G}$. Let $\mathscr{H} \leqq \mathscr{G}$. It is easy to see that the total number of $N$-sequences for $\mathscr{H}$ is

$$
f_{N}(\mathscr{H})=f\left(\mathscr{H} ; p^{N_{1}}, q^{N_{1}}, \cdots, p^{N_{r}}, q^{N_{r}}, \cdots\right) \text {. }
$$

Also, each $N$-sequence for $\mathscr{H}$ is generating $N$-sequence for a unique $\mathscr{K} \leqq \mathscr{H}$. Hence

and therefore

$$
f_{N}=\sum \phi_{N}
$$

$$
\phi_{N}(\mathscr{G})=\phi\left(\mathscr{G} ; p^{N_{1}}, q^{N_{1}}, \cdots, p^{N_{r}}, q^{N_{r}}, \cdots\right) .
$$

The fundamental property of the order polynomial is that

$$
f(\mathscr{G})=f(\mathscr{N}) f(\mathscr{G} / \mathscr{N})
$$

In fact, since

$$
G_{\kappa} N / N=G_{\kappa} / N_{\kappa} \quad(\kappa=i-1, i),
$$

we have the index formula

$$
\left|\Gamma_{i}: \Gamma_{i-1}\right|\left|N_{i}: N_{i-1}\right|=\left|G_{i}: G_{i-1}\right| \quad(\Gamma=G / N),
$$

of which (5.8) is an immediate consequence.

In view of (5.8), both (3.3) and theorem 1 carry over to the present case.

$$
\phi(\mathscr{G})=f(\Phi(\mathscr{G})) \phi(\mathscr{G} / \Phi(\mathscr{G})) .
$$

where $\Phi(\mathscr{G})$ is the subchain corresponding to $\Phi(G)$.

Theorem 3. If $\mathscr{N} \triangleleft \mathscr{G}$,

$$
f(\mathscr{N}) \phi(\mathscr{G} \mid \mathcal{N})=\sum_{\mathscr{H}=\mathscr{G}} \phi(\mathscr{H}) .
$$

It is not in general true that $\phi(\mathscr{G} / \mathscr{N}) \mid \phi(\mathscr{G})$. However, the following result does include the corollary to theorem 1 as a special case.

CoROLlaRY. If $\mathscr{N} \triangleleft \mathscr{G}$ and if $G_{\kappa-1} \leqq N \leqq G_{\kappa}$ for some $\kappa$, then $\phi(\mathscr{G} \mid \mathcal{N}) \mid \phi(\mathscr{G})$.

Proof. The proof of the corollary to theorem 1 shows that it is sufficient to prove the statement:

$$
\text { If } \mathscr{X} \mathscr{N}=\mathscr{G} \text {, then } \phi(\mathscr{K} \mid \mathscr{K} \wedge \mathscr{N})=\phi(\mathscr{G} / \mathscr{N}) \text {. }
$$

(5.12) is proved by showing that the natural homomorphism of $K / K \wedge N$ onto $K N / N=G / N$ maps $\mathscr{X} / \mathscr{K} \wedge \mathscr{N}$ onto $\mathscr{G} / \mathscr{N}$; i.e.

$$
K_{i} N=G_{i} N
$$$$
(0 \leqq i \leqq r) .
$$

By hypothesis, $G_{\kappa-1} \leqq N \leqq G_{k}$. If $i<\kappa$, clearly $K_{i} N=G_{i} N=N$. If $i \geqq \kappa, K_{i} N=\left(K \wedge G_{i}\right) N=(K N) \wedge G_{i}=G_{i}=G_{i} N$ because $G_{i} \geqq N$ and $K N=G$. This proves (5.13) and the corollary. 


\section{Chains in a $p$-Group}

In this section, $G$ is a $p$-group and $\mathscr{G}$ the corresponding chain (5.1) of formal length $r$. In order to simplify the notation, we observe the following conventions.

(a) Boldface letters (lower and upper case) stand for row vectors of length $r$ with non-negative integral components:

where

$$
\boldsymbol{m}=\left(m_{1}, \cdots, m_{r}\right), \quad \boldsymbol{M}=\left(M_{1}, \cdots, M_{r}\right), \cdots
$$

$$
m_{i} \geqq 0, M_{i} \geqq 0, \cdots \quad(1 \leqq i \leqq r) .
$$

(b) Corresponding lower and upper case vectors $\boldsymbol{m}, \boldsymbol{M}$ are related by:

$$
M_{i}=\sum_{j=i}^{r} m_{i}, \quad m_{i}=M_{i}-M_{i+1} \quad\left(M_{r+1}=0\right) \quad(1 \leqq i \leqq r) .
$$

Thus, the upper case vectors $M$ are those which satisfy:

$$
M_{1} \geqq M_{2} \geqq \cdots \geqq M_{r} \geqq M_{r+1}=0
$$

(c) Inequality $\boldsymbol{m} \geqq n$ means that $m_{i} \geqq n_{i}(1 \leqq i \leqq r)$.

Writing

where

$$
\begin{aligned}
\boldsymbol{m} & =\boldsymbol{m}(\mathscr{G})=\left(m_{1}, \cdots, m_{r}\right), \\
\boldsymbol{D} & =\boldsymbol{D}(\mathscr{G})=\left(D_{1}, \cdots, D_{r}\right),
\end{aligned}
$$

$$
\left.\begin{array}{l}
\left|G_{i}: G_{i-1}\right|=p^{m_{i}} \\
\left|\Gamma: \Gamma_{i-1}\right|=p^{D_{i}} \quad(\Gamma=G / \Phi(G))
\end{array}\right\} \quad(1 \leqq i \leqq r),
$$

we call $\mathscr{G}$ a $D$-generator chain of reduced order $m$. We first set down some fairly obvious properties of $\boldsymbol{m}, \boldsymbol{D}$.

(6.1) If $\mathscr{H}$ is a subchain or factor chain of $\mathscr{G}$,

$$
\boldsymbol{m}(\mathscr{H}) \leqq \boldsymbol{m}(\mathscr{G}) \text {. }
$$

$$
\begin{aligned}
\boldsymbol{m}(\Phi(\mathscr{G}))=\boldsymbol{m}(\mathscr{G})-\boldsymbol{d}(\mathscr{G}) ; \text { hence, if } \mathscr{H} \leqq \mathscr{G} \\
\boldsymbol{0} \leqq \boldsymbol{m}(\mathscr{H})-\boldsymbol{d}(\mathscr{H}) \leqq \boldsymbol{m}(\mathscr{G})-\boldsymbol{d}(\mathscr{G}) .
\end{aligned}
$$

(6.3) If $D_{i}=0$, then $M_{i}\left(=m_{i}+\cdots+m_{r}\right)=0$.

For if $D_{i}=0$, then $G_{i-1} \Phi(G)=G$ and so $G_{i-1}=G$; thus $p^{M_{i}}=\left|G: G_{i-1}\right|$ $=p^{0}$.

The Eulerian polynomial of $\mathscr{G}$ is calculated in much the same way as that of $G$, and we therefore omit the proof.

(6.4) If $\mathscr{G}$ is a $D$-generator chain of reduced order $\boldsymbol{m}$, then

$$
\phi\left(\mathscr{G} ; x_{1}, \cdots, x_{r}\right)=\prod_{i=1}^{r} x_{i}^{m_{i}-d_{t}} \prod_{\lambda_{i}=D_{i+1}}^{D_{i}-1}\left(x_{i}-p^{\lambda_{i}}\right) .
$$

The following immediate corollary justifies the term "D-generator chain". 
(6.5) Let $\mathscr{G}$ be a $\boldsymbol{D}$-generator chain and $N$ an upper case vector. Then $\phi_{N}(\mathscr{G})>0$ if, and only if, $N \geqq D$.

From now on we confine attention to regular chains in the sense of the following definition.

Definition. $\mathscr{G}$ is called regular if $\boldsymbol{m}(\mathscr{H})-\boldsymbol{D}(\mathscr{H})$ is upper case for each $\mathscr{H} \leqq \mathscr{G}$. If

$$
\boldsymbol{m}(\mathscr{H})-\boldsymbol{D}(\mathscr{H})=\boldsymbol{T}(\mathscr{H}),
$$

then the pair of vectors

$$
\sigma(\mathscr{H})=(\boldsymbol{t}(\mathscr{H}), \boldsymbol{D}(\mathscr{H}))
$$

is called the signature of $\mathscr{H}$.

Clearly, every subchain of a regular chain is regular (though the corresponding statement for factor chains is easily seen to be false). The Eulerian polynomial of a regular chain of signature $(\boldsymbol{t}, \boldsymbol{D})$ can be written in the form

$$
\phi\left(\mathscr{G} ; x_{1}, \cdots, x_{r}\right)=Y_{t, D}\left(1, y_{1}, \cdots, y_{r}\right)
$$

where

$$
y_{i}=x_{1} x_{2} \cdots x_{i} \quad(1 \leqq i \leqq r),
$$

We remark that $\boldsymbol{m}(\mathscr{H})-\boldsymbol{D}(\mathscr{H})$ is upper case if, and only if,

i.e.

$$
m_{i}(\mathscr{H})-d_{i}(\mathscr{H}) \geqq m_{i+1}(\mathscr{H}) \quad\left(1 \leqq i \leqq r ; m_{r+1}(\mathscr{H})=0\right)
$$

$$
m_{i}(\Phi(\mathscr{H})) \geqq m_{i+1}(\mathscr{H}) \quad(1 \leqq i \leqq r)
$$

Examples.

(1) If $r=1, \mathscr{G}$ is regular.

(2) Let $G$ be a regular group of exponent $p^{k}$ (P. Hall [5]). Then the elements of $G$ of order $\leqq p^{i}$ form a subgroup $\Omega_{i}(G)(i=0,1, \cdots)$. Consider the chain

$$
\mathscr{G}: 1=\Omega_{0}(G) \leqq \Omega_{1}(G) \leqq \cdots \leqq \Omega_{r}(G)=G,
$$

where $r \geqq k$. It is known that $m_{i}=m_{i}(\mathscr{G})$ is the number of elements of order $\geqq p^{i}$ in a basis of $G$. If $H \leqq G, H$ is regular and

$$
\mathscr{H}: 1=\Omega_{0}(H) \leqq \cdots \leqq \Omega_{r}(H)=H
$$

is the corresponding subchain of $\mathscr{G}$. We prove now that $\mathscr{G}$ is regular.

By the remarks above, it is sufficient to verify (6.8) for $\mathscr{G}$. Let $K$ be the subgroup of $G$ formed by the $p$-th powers of the elements of $G, \mathscr{K}$ the corresponding subchain of $\mathscr{G}$. It is known that the number of elements of order $\geqq p^{i}$ in a basis of $K$ is the number of elements of order $\geqq p^{i+1}$ in a basis of $G$. Therefore, since $K \leqq \Phi(G)$, we have 
as required.

$$
m_{i}(\Phi(\mathscr{G})) \geqq m_{i}(\mathscr{K})=m_{i+1}(\mathscr{G}),
$$

In general, if $G$ is a $p$-group in which the elements of order $\leqq p^{i}$ form a subgroup $\Omega_{i}(G)$ for $i=1,2, \cdots$, then we shall call a chain

$$
\mathscr{G}: \mathbf{1}=\Omega_{0}(G) \leqq \Omega_{1}(G) \leqq \cdots \leqq \Omega_{r}(G)=G \cdot
$$

an $\Omega$-series of $G$. The example of the quaternion group shows that an $\Omega$ series need not be regular.

(3) If the regular group $G$ in (2) is abelian then $K=\Phi(G)$ and so

$$
m_{i}(\Phi(\mathscr{G}))=m_{i+1}(\mathscr{G}) \quad(1 \leqq i \leqq r) ;
$$

thus the signature of $\mathscr{G}$ (and of each subchain) has the form $(\boldsymbol{O}, \boldsymbol{D}(\mathscr{G}))$. Following P. Delsarte ([2]), we shall call $G$ the abelian group of signature $\boldsymbol{D}(\mathscr{G})$.

In general, if $\mathscr{G}$ is a regular chain in the $p$-group $G$ and if $\mathscr{H} \leqq \mathscr{G}$ has signature $(\boldsymbol{O}, \boldsymbol{D})$, then we shall say that the subgroup $H$ corresponding to $\mathscr{H}$ has zero type with respect to $\mathscr{G}$. Since the vector $\boldsymbol{m}(\mathscr{H})-\boldsymbol{D}(\mathscr{H})$ is upper case, $H$ has zero type if, and only if, $m_{1}(\mathscr{H})=D_{1}(\mathscr{H})$. Simple examples show that a regular group may have zero type with respect to its $\Omega$-series and yet not be lattice-isomorphic to an abelian group.

(4) Let

$$
\mathscr{G}^{*}: 1=G_{0}^{*} \leqq \cdots \leqq G_{s}^{*}=G
$$

be any regular chain. Form a new chain

by taking

$$
\mathscr{G}: 1=G_{0} \leqq \cdots \leqq G_{r}=G
$$

$$
G_{i}=G_{N_{1}+N_{2}+\cdots+N_{i}}^{*} \quad(1 \leqq i \leqq r),
$$

where $N=\left(N_{1}, \cdots, N_{r}\right)$ is upper case. It is easily verified (using (6.8)) that $\mathscr{G}$ is regular.

The results which follow deal with the property of having zero type. (6.9) Let $\mathscr{G}$ be a regular chain in the p-group $G$. Then $G_{i}$ contains every element of $G$ of order $\leqq p^{i}(i=1,2, \cdots)$.

Proof. Let $H$ be a cyclic subgroup of $G$ of order $p^{k} \leqq p^{i}, \mathscr{H}$ the corresponding subchain of $\mathscr{G}$. Write

Then

$$
m_{j}=m_{j}(\mathscr{H}), \quad D_{j}=D_{j}(\mathscr{H}) .
$$

$$
m_{1} \geqq m_{\mathrm{u}} \geqq \cdots \text { and } \sum m_{j}=k \text {, }
$$

whence $m_{k+1}=m_{k+2}=\cdots=0$. Thus, $H \leqq G_{k} \leqq G_{i}$.

(6.11) Let $\mathscr{G}$ be a regular chain in the p-group $G$. Then $\mathscr{G}$ is an $\Omega$-series of $G$ if, and only if, each cyclic subgroup of $G$ has zero type with respect to $\mathscr{G}$. 
Proof. Suppose that the cyclic subgroup $H$ of $G$ has zero type. In the notation of the previous proof,

$$
m_{1}-D_{1}=0, \quad D_{1}=1,
$$

so that, by $(6.10)$,

$$
m_{1}=m_{2}=\cdots=m_{k}=1 .
$$

Hence $H$ is a subgroup of $G_{k}$ but not of $G_{k-1}$. It follows that if every cyclic subgroup of $G$ has zero type then $G_{i}-G_{i-1}$ is the set of elements of $G$ of order $p^{i}(i=1,2, \cdots)$, i.e. $\mathscr{G}$ is an $\Omega$-series of $G$. The converse is easily proved by reversing the steps of the argument.

(6.12) Let $G$ be a regular chain in the p-group $G$ and suppose that every subgroup of $G$ which can be generated by 2 elements has zero type with respect to $G$. Then, if $p>3$, or if $G$ is regular, $G$ is lattice-isomorphic to an abelian group and $\mathscr{G}$ is an $\Omega$-series of $G$.

Proof. Since every regular 2-group is abelian (Kemhadze [12]), we assume that $p>2$. We first prove, by induction on the order of $G$, that any two cyclic subgroups of $G$ permute. We know already (by (6.11)) that $\mathscr{G}$ is an $\Omega$-series of $G$. This implies, in particular, that each $G_{i}$ is a normal subgroup of $G$.

Suppose that the cyclic subgroups $X=\{x\}, Y=\{y\}$ did not permute. By induction, $\{X, Y\}=G$ and so, in particular, $D_{1}(\mathscr{G})=2$. Since $G$ has zero type, $m_{1}(\mathscr{G})=2$ i.e.

$$
\left|G_{1}\right|=p^{2} .
$$

Write $|G|=p^{a},|X|=p^{\lambda},|Y|=p^{\mu}$. If $\lambda$ (say) were 1 , we should have $X Y=Y$ or $Y G_{1}$, contrary to the assumption that $X Y$ is not a subgroup; hence $\lambda>1, \mu>1$.

It is a straightforward matter to verify that the chain

$$
\mathscr{G}^{\prime}: 1=G_{1} / G_{1}<G_{2} / G_{1}<\cdots<G_{r} / G_{1}=G / G_{1}
$$

is regular and that every subgroup of $G / G_{1}$ which can be generated by 2 elements has zero type with respect to $\mathscr{G}^{\prime}$. By induction, $\left\{x G_{1}\right\}$ and $\left\{y G_{1}\right\}$ permute, so that

$$
X Y G_{1}=G .
$$

Consider now the subgroups

$$
X^{*}=\left\{x^{\dot{p}}\right\}, \quad Y^{*}=\left\{y^{p}\right\}, \quad H=\left\{X^{*}, Y\right\}, \quad K=\left\{X, Y^{*}\right\} .
$$

Since $X^{*}, Y^{*}$ are subgroups of $\Phi(G), H, K$ are proper subgroups of $G$. By induction, $H=X^{*} Y, K=X Y^{*}$. If $H_{1}=G_{1}$ then, by (6.14), $X Y=G$, contrary to the assumption that $X, Y$ do not permute. Hence $\left|H_{1}\right|=p$ 
and similarly $\left|K_{1}\right|=p$. Since $H, K$ have zero type, they must be cyclic groups. Thus, $X^{*}=Y^{*}=X \wedge Y$. It follows that $\lambda=\mu,|X Y|=p^{\lambda+1}$, and from (6.14) that $|X Y|=p^{a-1}$. Thus $\lambda=a-2$.

It is now clear that $X^{*}$ is a central normal subgroup of $G$ of index $p^{3}$ and order $\geqq p$. The factor group $G / X^{*}$ is non-commutative and is generated by the elements $x X^{*}, y Y^{*}$ of order $p$; it is therefore the non-commutative group of order $p^{3}$ and exponent $p$. Writing $u=y x y^{-1} x^{-1}$, we have:

$$
y u y^{-1} u^{-1}=c, \quad x u x^{-1} u^{-1}=d \quad\left(c, d \in X^{*}\right), \quad\left|\left\{u X^{*}\right\}\right|=p,
$$

(6.15) $x^{\alpha} y^{\beta} \in\left\{u X^{*}\right\}$ if, and only if, $\alpha \equiv \beta \equiv 0(\bmod p)$.

Since $y^{p}, x^{p}$ are in the centre of $G, c^{p}=d^{p}=c^{\left(\frac{p}{2}\right)} u^{p}=1$. Therefore, since $p>2, u^{p}=1$.

It follows from the above and (6.13) that

$$
G_{1}=\left\{u, x^{p^{a-s}}\right\}
$$

On the other hand, a direct calculation shows that

$$
(x y)^{p}=u^{\left(\frac{p}{2}\right)} x^{p} y^{p} c^{\left(\frac{p}{3}\right)} d^{\left(\frac{p}{2}\right)+2\left(\frac{p}{3}\right)}
$$

and therefore, if $p>3$,

$$
(x y)^{p}=x^{p} y^{p} .
$$

This also holds if $p=3$, for then $G$ is regular and so

$$
(x y)^{3}=x^{3} y^{3} u^{3 \alpha} c^{3 \beta} d^{3 \gamma}=x^{3} y^{3} \text {. }
$$

Hence, in all cases, for a suitable choice of the generators $x, y$, we have $x y \in G_{1}$. This contradiction to (6.15) and (6.16) establishes our result.

It now follows that any two subgroups of $G$ permute and thence that the lattice of subgroups of $G$ is modular. Since $p>2, G$ is lattice-isomorphic to an abelian group (M. Suzuki [8]).

Q.E.D.

(6.12) is not true for non-regular 2- and 3-groups, as the $\Omega$-series in the following groups show t:

$$
\begin{aligned}
& \text { (6.17) } G=\{x, y, u\} \text { of order } 3^{4} ; x^{9}=u^{3}=x^{3} y^{3}=[x, u]=1, \\
& {[y, x]=u,[y, u]=y^{3} .} \\
& \text { (6.18) } G=\{x, y\} \text { of order } 2^{r+2} \geqq 2^{4} ; x^{2^{r}}=y^{4}=1, \quad[y, x]=y^{2} . \\
& \text { (6.19) } G=\{x, y\} \text { of order } 2^{r+2} \geqq 2^{6} ; x^{2^{r}}=y^{4}=1, \quad[y, x]=y^{2} x^{2^{r-2}} .
\end{aligned}
$$

These examples are all "minimal" in the sense that every proper subgroup of $G$ is lattice-isomorphic to an abelian group. $G$ itself is not lattice-isomorphic to an abelian group because (in each case) $G /\left\{x^{p}\right\}$ is the group of order $p^{3}$ generated by 2 elements of order $p$.

We end this section by deriving the analogue of (4.5)' for the polynomials $Y_{t, D}$ (cf. (6.7)).

$+[s, t]$ stands for the commutator $s t s^{-1} t^{-1}$. 
Lemma. Let $d, D, s, t$ be integers $\geqq 0$. Then

$$
x^{t} \prod_{\lambda=0}^{d-1}\left(x-y p^{D+\lambda}\right)=\sum_{i=0}^{\min (d, s)} \sum_{j=0}^{t} \theta(d, D, s, t ; i, j) y^{t-s+i} \prod_{\mu=0}^{d+j-i-1}\left(x-y p^{D+s+\mu}\right),
$$

where

$$
\theta(d, D, s, t ; i, j)=\omega(s, i) \omega(d, i) \omega(t, j) X_{i}\left(p^{i}\right) p^{(t-j)(D+d+s-i)+i D} .
$$

(For the notation $\omega(k, l), X_{k}(x)$ see $(4.1)^{\prime},(4.3)^{\prime}$.)

Proof. It is easily proved by induction that

$$
X_{d}(z) X_{s}(z)=\sum_{i=0}^{\min (d, s)} \omega(d, i) \omega(s, i) X_{i}\left(p^{i}\right) X_{d+s-i}(z)
$$

Combining this formula with (4.5)', we get

$$
z^{t} X_{d}(z) X_{s}(z)=\sum_{i=0}^{\min (d, s)} \omega(d, i) \omega(s, i) X_{i}\left(p^{i}\right) \sum_{j=0}^{t} \omega(t, j) p^{(t-j)(d+z-i)} X_{d+s+j-i}(z) .
$$

Finally, dividing through by $X_{s}(z)$, then putting $z=x y^{-1} p^{-D}$ and simplifying, we get (6.20).

THEOREM 4.

$$
Y_{t, D}\left(z_{0}, \cdots, z_{r}\right)=\sum_{U}\left(\sum_{i} \prod_{k=1} \beta_{\kappa}(i, U)\right) z_{0}^{T_{1}+D_{1}-U_{1}} Y_{0, U}\left(z_{0}, \cdots, z_{r}\right),
$$

where (cf. (6.21))

$\beta_{\kappa}(I, U)=\theta\left(d_{\kappa}, D_{\kappa+1}, U_{\kappa+1}-D_{\kappa+1}, T_{\kappa}+D_{\kappa+1}-U_{\kappa+1} ; i_{\kappa}, u_{\kappa}-d_{\kappa}+i_{\kappa}\right)(1 \leqq \kappa \leqq r)$,

and where summation is over the vectors $U, I$ such that

$$
D \leqq U \leqq T+D,
$$

$$
d_{\kappa}-u_{\kappa} \leqq i_{\kappa} \leqq \min \left(d_{\kappa}, U_{\kappa+1}-D_{\kappa+1}, T_{\kappa}+D_{\kappa}-U_{\kappa}\right) \quad(1 \leqq \kappa \leqq r) .
$$

$\left(D, U, \cdots\right.$ are the upper case vectors corresponding to $d, u, \cdots ; D_{r+1}$, $U_{r+1}, \cdots$ are taken to be zero.)

Proof. Let us change the variables of summation from $i, U$ to $i$ and $\boldsymbol{j}=\boldsymbol{u}+\boldsymbol{i}-\boldsymbol{d}$. Then (6.22) becomes the final formula $P_{1}$ of a series $P_{r+1}, P_{r}, \cdots, P_{1}$ defined as follows: $P_{r+1}$ is the trivial formula $1=1 ; P_{\rho}$ $(1 \leqq \rho \leqq r)$ is the formula

where

$$
\prod_{\kappa=\rho}^{r} z_{\kappa}^{t_{\kappa}} Y_{\kappa}=\sum_{i, j} z_{\rho-1}^{T_{\rho}-J_{\rho}+I_{\rho}} \prod_{\kappa=\rho}^{r} Z_{\kappa}(i, j)
$$

$$
\begin{aligned}
Y_{\kappa} & =\prod_{\lambda=D_{k+1}}^{D_{k-1}}\left(z_{\kappa}-z_{\kappa-1} p^{\lambda}\right), \\
Z_{k}(i, j) & \left.=\beta_{\kappa} \prod_{\lambda=D_{k+1}+J_{k+1}-I_{k+1}} \prod_{k}-I_{k-1}-z_{k-1} p^{\lambda}\right),
\end{aligned}
$$


and where summation is over the indices $i_{\kappa}, j_{\kappa}(\rho \leqq \kappa \leqq r)$ which satisfy

$$
\left.\begin{array}{l}
0 \leqq i_{\kappa} \leqq \min \left(d_{\kappa}, J_{\kappa+1}-I_{\kappa+1}\right), \\
0 \leqq j_{\kappa} \leqq T_{\kappa}-J_{\kappa+1}+I_{\kappa+1}
\end{array}\right\} \quad(\rho \leqq \kappa \leqq r) .
$$

Now, by the lemma,

$$
z_{\rho-1}^{t_{\rho-1}+T_{\rho}-J_{\rho}+I_{\rho}} Y_{\rho-1}=\sum z_{\rho-2}^{T_{\rho-1}-J_{\rho-1}+I_{\rho-1}} Z_{\rho-1}(i, j),
$$

where summation is over the indices $i_{\rho-1}, j_{\rho-1}$ such that

$$
\begin{aligned}
& 0 \leqq i_{\rho-1} \leqq \min \left(d_{\rho-1}, J_{\rho}-I_{\rho}\right), \\
& 0 \leqq j_{\rho-1} \leqq T_{\rho-1}-J_{\rho}+I_{\rho} .
\end{aligned}
$$

Multiply both sides of $P_{\rho}$ by $z_{\rho-1}^{t \rho-1} Y_{\rho-1}$, and using (6.25), we get $P_{\rho-1}$. The theorem follows by induction.

COROLlary. Let $G$ be the abelian p-group of (Delsarte) signature $T$. Then the number of subgroups of $G$ of signature $U(\leqq T)$ is

$$
\Omega(\boldsymbol{T}, \boldsymbol{U})=\prod_{\kappa=1}^{r} \omega\left(T_{\kappa}-U_{\kappa+1}, u_{\kappa}\right) p^{\left(\boldsymbol{T}_{\kappa}-U_{\kappa}\right) U_{\kappa+1}}
$$

In fact, taking $D=O$ in (6.22), we get

$$
Y_{t, 0}=\sum_{U \leqq T} \Omega(T, U) Y_{0, U}
$$

which is essentially the Euler summation formula for the $\Omega$-series of $G$.

Formulae equivalent to (6.26) have been given by Delsarte [2], Kinosita [6], Yeh [10].

\section{Numbers of Subgroups of a p-Group}

We are now in a position to generalize the results of $\S 4$. Throughout the present section,

$$
\mathscr{G}: 1=G_{0} \leqq G_{1} \leqq \cdots \leqq G_{r}=G
$$

is a regular chain, of formal length $r$, in the p-group $G . m=m(\mathscr{G})$ is the reduced order of $\mathscr{G} . B$ is the abelian group of signature $\boldsymbol{m}$, and

$$
\mathscr{B}: 1=B_{0} \leqq B_{1} \leqq \cdots \leqq B_{r}=B
$$

an $\Omega$-series of $B$. Since $\boldsymbol{m}(\mathscr{G})=\boldsymbol{m}(\mathscr{B})$, we have

$$
f(\mathscr{G})=f(\mathscr{B}) .
$$

THEOREM 5. Let $N_{\gamma, \Delta}=N_{\gamma, \Delta}(\mathscr{G})$ denote the number of subchains of $\mathscr{G}$ of signature $(\gamma, 4)$. Then

$$
\Omega(\boldsymbol{m}, \boldsymbol{U})=\sum_{\boldsymbol{\Delta} \leqq U \leqq \Gamma+\Delta} \beta(\gamma, \Delta ; \boldsymbol{U}) N_{\gamma, \Delta} \quad(\boldsymbol{U} \leqq \boldsymbol{m}),
$$


where $\Omega(\boldsymbol{m}, \boldsymbol{U})$ is given by (6.26) and $\beta(\gamma, \Delta ; \boldsymbol{U})$ is the coefficient of

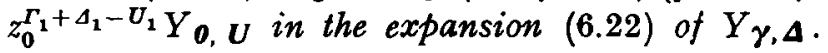

Proof. Let us denote the Eulerian polynomial (6.6) by $\bar{Y}_{t, D}$. Then, by the Euler summation formulae for $\mathscr{G}, \mathscr{B}$,

$$
\begin{aligned}
& f(\mathscr{G})=\sum_{\gamma, \Delta} N_{\gamma, \Delta} \bar{Y}_{\gamma, \Delta}, \\
& f(\mathscr{B})=\sum_{\boldsymbol{U}} \Omega(\boldsymbol{m}, \boldsymbol{U}) \bar{Y}_{\boldsymbol{O}, U},
\end{aligned}
$$

and so, by (7.3) and theorem 4,

$$
\sum \Omega(\boldsymbol{m}, \boldsymbol{U}) \vec{Y}_{\boldsymbol{0}, \boldsymbol{U}}=\sum N_{\boldsymbol{\gamma}, \Delta} \beta(\boldsymbol{\gamma}, \Delta ; \boldsymbol{U}) \overline{\mathbf{Y}}_{\mathbf{0}, \boldsymbol{U}} .
$$

Since the $\bar{Y}_{\boldsymbol{O}, U}$ are linearly independent, (7.4) follows.

Q.E.D.

Lemma. $\beta=\beta(\gamma, \boldsymbol{\Delta} ; \boldsymbol{U})$ is a positive integer whenever $\boldsymbol{\Gamma} \leqq \boldsymbol{U} \leqq \boldsymbol{\Gamma}+\boldsymbol{\Delta}$, If $\boldsymbol{U}=\boldsymbol{\Gamma}+\Delta, \beta=1$. If $\boldsymbol{U} \neq \boldsymbol{\Gamma}+\Delta($ and $\boldsymbol{\Gamma} \leqq U \leqq \Gamma+\Delta)$, either $N_{\gamma, \Delta}=0$ or $\beta \equiv 0(\bmod p)$.

Proof. The first two statements are seen by inspection. To prove the third, we show that if $\boldsymbol{U} \neq \boldsymbol{\Gamma}+\boldsymbol{\Delta}$ and $N_{\gamma, \boldsymbol{\Delta}} \neq 0$ then each term $\beta(i)=\prod_{\kappa} \beta_{\kappa}(i)$ in the sum $\beta=\sum_{i} \beta(i)$ is divisible by $p$ (cf. (6.22)).

Suppose, contrary to our assertion, that $\beta(i) \neq 0(\bmod p)$ for some value of $\boldsymbol{i}$. By (6.21) and (6.22),

$$
\left.\begin{array}{l}
\frac{1}{2} i_{\kappa}\left(i_{\kappa}-1\right)=0, \\
\left(\Gamma_{\kappa}+\Delta_{\kappa}-U_{\kappa}-i_{\kappa}\right)\left(\delta_{\kappa}+U_{\kappa+1}-i_{\kappa}\right)=0, \\
i_{\kappa} \Delta_{\kappa+1}=0
\end{array}\right\} \quad(1 \leqq \kappa \leqq r) .
$$

By assumption, $\Gamma_{\lambda}+\Delta_{\lambda}-U_{\lambda} \neq 0$ for some value $\lambda$. By $(7.4)^{\prime}, i_{\lambda}=1$ or 0 . If $i_{\lambda}=1$, then, by $(7.4)^{\prime}, \Delta_{\lambda+1}=0$. By (6.3) and since $N_{\gamma, 4} \neq 0, \Gamma_{\lambda+1}+$ $\Delta_{\lambda+1}=0$. Therefore, since $\boldsymbol{U} \leqq \Gamma+\Delta, U_{\lambda+1}=0$. This is impossible for, by (6.24), $i_{\lambda} \leqq \Delta_{\lambda+1}+U_{\lambda+1}$. Hence $i_{\lambda}=0$. By (7.4)', $\delta_{\lambda}=U_{\lambda+1}=0$. Since $\Delta \leqq U, \Delta_{\lambda+1}=0$ and so $\Delta_{\lambda}=\delta_{\lambda}+\Delta_{\lambda+1}=0$. Arguing as before, we get $U_{\lambda}=\Gamma_{\lambda}+\Delta_{\lambda}=0$, contrary to the assumption that $\Gamma_{\lambda}+\Delta_{\lambda}-U_{\lambda} \neq 0$. This establishes the lemma.

Write

$$
\begin{aligned}
& N_{U}(\mathscr{G})=\sum_{\Delta \leqq U \leqq \Gamma+\Delta} N_{\gamma, \Delta}(\mathscr{G}), \\
& n_{U}(\mathscr{G})=\sum_{U=\Gamma+\Delta} N_{\gamma, \Delta}(\mathscr{G}), \\
& N_{k}(G)=\sum_{\Delta_{1} \leqq k \leqq \Sigma\left(\Gamma_{i}+\Delta_{i}\right)} N_{\gamma, \Delta}(\mathscr{G}), \\
& n_{k}(G)=\sum_{k=\Sigma\left(\Gamma_{i}+\Delta_{i}\right)} N_{\gamma, \Delta}(\mathscr{G}), \\
& n(G)=\sum_{k} n_{k}(G) .
\end{aligned}
$$


In words: $N_{U}$ is the number of subchains which have a generating $U$ sequence and reduced order $\geqq U ; n_{U}$ is the number of subchains of reduced order $U ; N_{k}, n_{k}$ are the functions already considered in $\S 4 ; n(G)$ is the total number of subgroups of $G$. Clearly,

$$
N_{U}(\mathscr{B})=n_{U}(\mathscr{B})=\Omega(\boldsymbol{m}, \boldsymbol{U}) .
$$

The following two results are immediate consequences of theorem 5 and the lemma.

(7.5) Either $n_{U}(\mathscr{G})<N_{U}(\mathscr{G})<\Omega(\boldsymbol{m}, \boldsymbol{U})$ or $n_{U}(\mathscr{G})=N_{U}(\mathscr{G})=\Omega(\boldsymbol{m}, \boldsymbol{U})$.

(7.6) $n_{U}(\mathscr{G}) \equiv \Omega(m, U) \quad(\bmod p)$.

The congruence class of $\Omega(\boldsymbol{m}, \boldsymbol{U})$ modulo $p$ is easily determined. Suppose that $U_{s} \neq 0$ but $U_{\lambda}=0$ for all $\lambda>s$. Then, by $(6.26), \Omega \equiv 1(\bmod p)$ if $m_{\lambda}=U_{\lambda}$ for $\lambda=1, \cdots, s-1$, and $\Omega \equiv 0(\bmod p)$ otherwise. Hence we hâve

$n_{U}(\mathscr{G}) \equiv 1$ or $0(\bmod p)$ according as $U$ is, or is not, the signature of a subgroup of $B$ which lies between two consecutive members of its $\Omega$ series.

(7.8) $N_{k}(G) \leqq N_{k}(B)$, with equality if, and only if, every subgroup of $G$ which has order $>p^{k}$ and can be generated by $k$ elements has zero type with respect to $\mathscr{G}(C f . \S 6$, example (3)).

Proof. By the definition of $N_{k}$ and theorem 5, we have

$$
\begin{aligned}
& N_{k}(G)=\sum_{\Delta_{1} \leqq k \leqq \Sigma\left(\Gamma_{i}+\Delta_{i}\right)} N_{\gamma, \Delta}(\mathscr{G}), \\
& N_{k}(B)=\sum_{U_{1} \leqq k \leqq \Sigma U_{i}} \Omega(\boldsymbol{m}, \boldsymbol{U}) \\
& =\sum_{U_{1} \leqq k \leqq \Sigma U_{i} \Delta \leqq U \leqq \Gamma+\Delta} \beta(\gamma, \Delta ; U) N_{\gamma, \Delta}(\mathscr{G}) .
\end{aligned}
$$

Now, if the vectors $\gamma, \Delta$ satisfy $\Delta_{1} \leqq k \leqq \sum\left(\Gamma_{i}+\Delta_{i}\right)$, then the vector $V$ defined by

$$
V_{i}=\min \left(k, \Gamma_{i}+\Delta_{i}\right) \quad(1 \leqq i \leqq r)
$$

is upper case and satisfies

$$
V_{1} \leqq k \leqq V_{i}, \quad \Delta \leqq V \leqq \Gamma+\Delta .
$$

In view of (7.9), $N_{k}(G) \leqq N_{k}(B)$ with equality if, and only if, the conditions

$$
\Delta_{1} \leqq k \leqq \sum\left(\Gamma_{i}+\Delta_{i}\right), \quad N_{\gamma, \Delta}(\mathscr{G}) \neq 0,
$$

imply that

(a) (7.10) has a unique solution $V$;

(b) $\beta(\gamma, \Delta ; V)=1$, i.e., by the lemma, $V=\Gamma+\Delta$. 
We remark now that (7.11) and either of the two conditions

$$
\sum\left(\Gamma_{i}+\Delta_{i}\right)=k, \quad \boldsymbol{\Gamma}=\boldsymbol{0},
$$

imply (a) and (b). On the other hand, if neither of the two conditions (7.12) holds then (7.11) does not imply (a) and (b); for it is easy to see that there exists an upper case vector $\boldsymbol{R}$ such that

$$
\Delta \leqq R \leqq \Gamma+\Delta, \quad \sum R_{i}=\sum\left(\Gamma_{i}+\Delta_{i}\right)-1,
$$

and then the equations

$$
V_{i}=\min \left(k, R_{i}\right)
$$$$
(1 \leqq i \leqq r)
$$

define a solution $V$ of (7.10) distinct from $\boldsymbol{\Gamma}+\boldsymbol{\Delta}$.

It follows that $N_{k}(G)=N_{k}(B)$ if, and only if, the conditions

$$
\Delta_{1} \leqq k<\sum\left(\Gamma_{i}+\Delta_{i}\right), \quad \Gamma \neq 0,
$$

imply that $N_{\gamma, \Delta}(\mathscr{G})=0$. This is precisely the condition for equality stated in (7.8).

(7.13) $n_{k}(G) \leqq n_{k}(B)$, with equality if, and only if, every subgroup $H$ of $G$ which satisfies $\sum D_{i}(\mathscr{H}) \leqq k$ has order $\leqq p^{k}$.

Thus, the conditions for equality in (7.8) and (7.13) are

$$
\begin{aligned}
& \Delta_{1} \leqq k<\sum\left(\Gamma_{i}+\Delta_{i}\right) \text { and } \Gamma \neq 0 \Rightarrow N_{\gamma, \Delta}=0, \\
& \sum \Delta_{i} \leqq k<\sum\left(\Gamma_{i}+\Delta_{i}\right) \Rightarrow N_{\gamma, \Delta}=0,
\end{aligned}
$$

respectively. Obviously, the equality $N_{k}(G)=N_{k}(B)$ implies the equality $n_{k}(G)=n_{k}(B)$. We omit the proof of (7.13), which is similar to that of (7.8).

(7.14) The number of subgroups of $G$ which can be generated by $k$ elements cannot exceed the number of subgroups of $B$ which can be generated by $k$ elements. Each of the following is a necessary and sufficient condition for equality:

(i) $n_{\lambda}(G)=n_{\lambda}(B)(\lambda=1, \cdots, k-1)$ and $N_{k}(G)=N_{k}(B)$;

(ii) every subgroup of $G$ which can be generated by $k$ elements has zero type with respect to $\mathscr{G}$.

This follows easily from the results above and the fact that the number of subgroups of $G$ which can be generated by $k$ elements is $\sum_{\lambda=0}^{k-1} n_{\lambda}(G)+N_{k}(G)$. The next result is an immediate consequence.

(7.15) The following conditions are equivalent:

(i) $n_{k}(G)=n_{k}(B)$ for all $k$;

(ii) $N_{k}(G)=N_{k}(B)$ for all $k$;

(iii) $n(G)=n(B)$;

(iv) every subgroup of $G$ has zero type with respect to $\mathscr{G}$.

Together with (6.12), these results give 
(7.16) Suppose that $p>3$ or that $G$ is regular. Then the following conditions are equivalent:

(i) the total number of subgroups of $G$ equals the total number of subgroups of $B$;

(ii) the number of subgroups of $G$ which can be generated by 2 elements equals the number of subgroups of $B$ which can be generated by 2 elements;

(iii) $G$ is an $\Omega$-series of $G$ and $G$ is lattice-isomorphic to $B$.

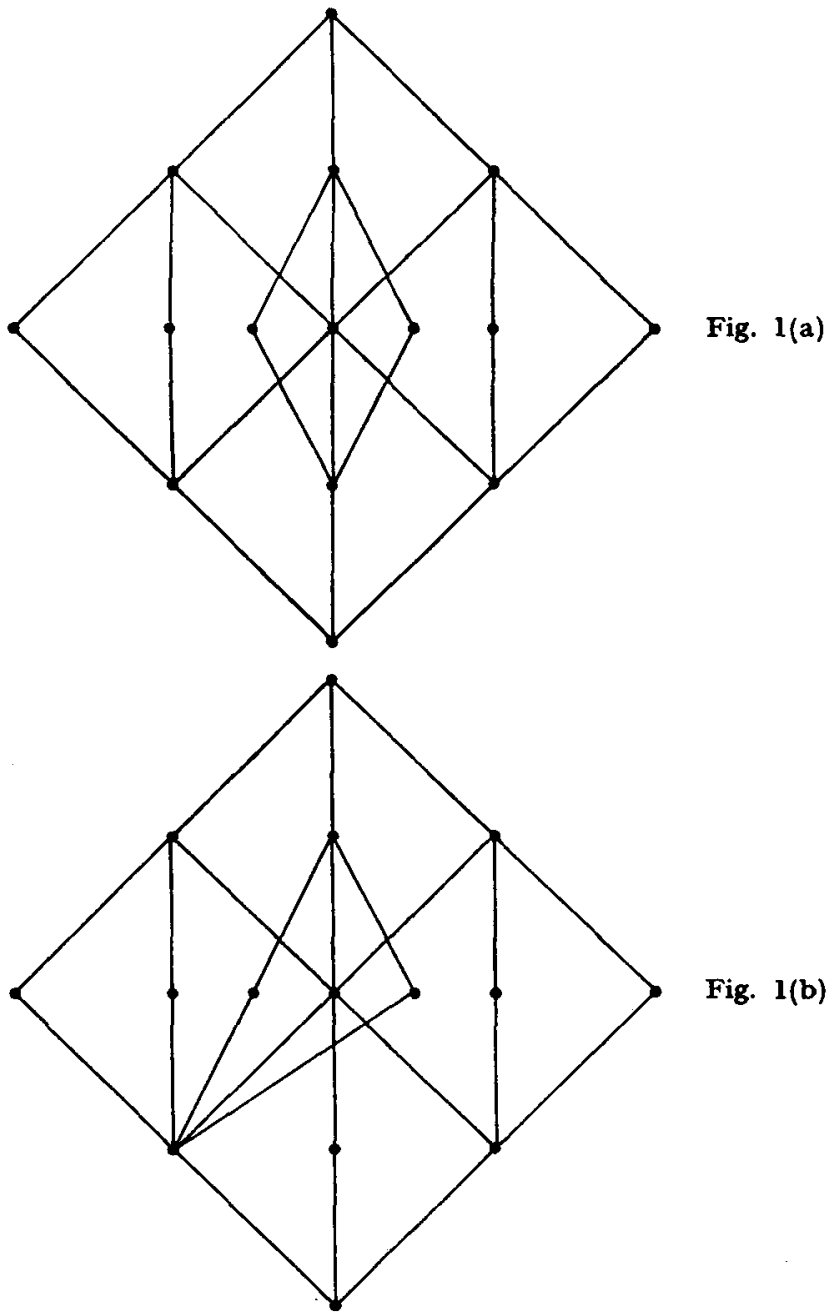

The subgroup lattices in figures 1,2 show that (7.16) (i) does not imply (7.16) (iii) when $G$ is a non-regular 2- or 3-group. Fig. 1a is the Abelian group of type $\left(2^{2}, 2^{2}\right)$, fig. $1 b$ the group $(6.18)$ of order $2^{4}$. Fig. $2 a$ is the Abelian group of type $\left(3^{2}, 3^{2}\right)$, fig. $2 b$ the group (6.17) of order $3^{4}$. 


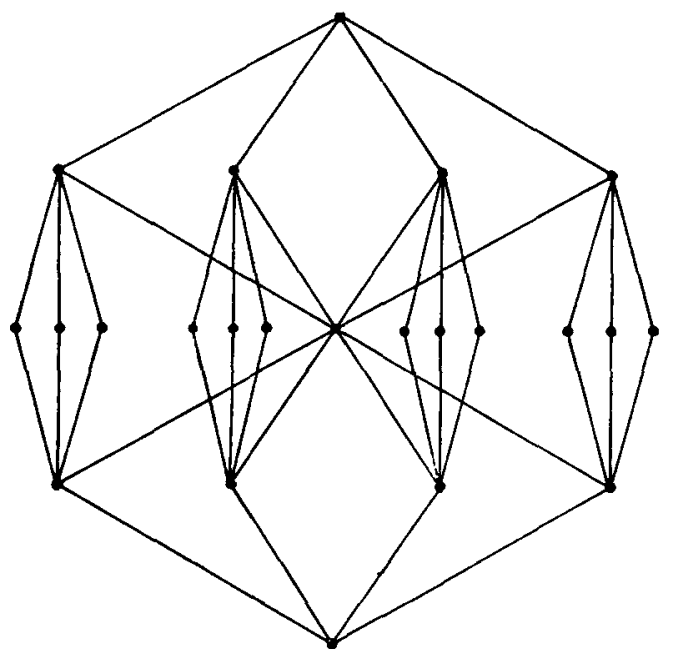

Fig. (2a)

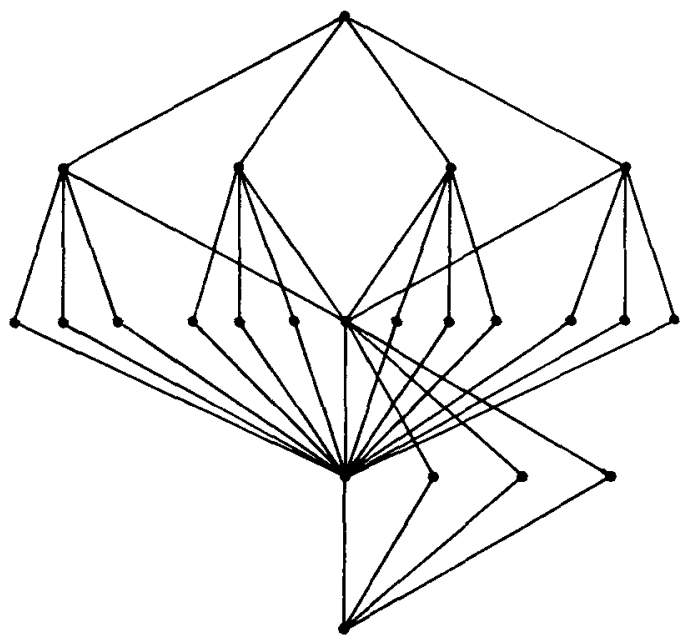

Fig. 2(b)

\section{The Eulerian Polynomial of a Group of Composite Order}

We end with some remarks on soluble and $p$-soluble groups. Let $G$ be a group of order $p^{a} q^{b} \cdots$, where $p, q, \cdots$ are distinct primes, and let $x, y, \cdots$ be the variables in the Eulerian polynomial corresponding to $p, q, \cdots$ respectively. Let $N$ be a normal subgroup of $G$ of order $n$. By theorem 1 and induction on the group order

$$
\phi(G)=\phi(G / N) \chi(x, y, \cdots),
$$

where $\chi$ involves only those variables which correspond to prime divisors of $n$. Hence, if $G$ be $p$-soluble, 


$$
\phi(G)=\rho(x) \sigma(y, z, \cdots),
$$

and if $G$ be soluble,

$$
\phi(G)=\rho(x) \sigma(y) \tau(z) \cdots .
$$

The last result is due to $W$. Gaschütz ([3]).

Gaschütz's method for determining the factors $\rho, \sigma, \cdots$ when $G$ is soluble applies equally well to determine the factor $\rho$ when $G$ is $p$-soluble. Suppose that $G$ is $p$-soluble and consider the factors in a given chief series for $G$. Such a factor $H / K$ is said to be complemented when $H / K$ is complemented in $G / K$. Now, since $G$ is $p$-soluble, either $|H / K|$ is prime to $p$ or $H / K$ is an elementary abelian $p$-group. In the latter case, $H / K$ may be regarded, in the usual way, as an irreducible $G$-module; as such, it is a vector space over its field, $E$, of endomorphisms. If $|E|=p^{e}$ and $\operatorname{dim}_{E}(H / K)=f$, then $|H| K \mid$ $=p^{e f}$.

Under the relation of $G$-module isomorphism, the complemented chief factors of $p$-power order fall into a certain number of equivalence classes, say $C_{0}, C_{1}, \cdots, C_{r}$. Suppose that $C_{0}$ consists of the (complemented) factors on which $G$ acts trivially. Suppose also that $C_{i}$ has $k_{i}$ members and that the values of $e, f$ corresponding to these members are $e_{i}, f_{i}(i=0,1, \cdots, r)$. Suppose finally that the product of the orders of the uncomplemented chief factors of $p$-power order is $p^{s}$. Then

$$
\rho(x)=x^{s}\left(\prod_{j=0}^{k_{0}-1}\left(x-p^{j}\right)\right) \prod_{i=1}^{r} \prod_{\lambda_{i}=0}^{k_{i} \rightarrow 1}\left(x^{e_{i} f_{i}}-p^{\boldsymbol{e}_{i}\left(f_{i}+\lambda_{i}\right)}\right) .
$$

It is easily proved that in any finite group the index of each maximal subgroup divides the order of at least one chief factor. Thus, in a $p$-soluble group, the index of each maximal subgroup is either a power of $p$ or prime to $p$. Let $m_{p}(G)$ denote the number of maximal subgroups of $G$ of $p$-power index $(>1)$, and write

$\mu_{0}=\left(p^{k_{0}}-1\right) /(p-1), \quad \mu_{i}=\left(p^{e_{i}\left(f_{i}+k_{i}\right)}-p^{e_{i} f_{i}}\right) /\left(p^{e_{i}}-1\right) \quad(i=1, \cdots, r)$.

Gaschütz's method shows that

$$
m_{p}(G)=\sum_{i=0}^{+} \mu_{i}
$$

and, more precisely, that the number of maximal subgroups of index $p^{k}$ is

$$
\sum_{i, f_{i}=k} \mu_{i}
$$

Now, it is easily verified that

Therefore, since

$$
\mu_{i} \leqq p\left(p^{e_{i} t_{i} k_{i}}-1\right) /(p-1) \quad(0 \leqq i \leqq r) .
$$

$$
\sum_{0}^{r} e_{i} f_{i} k_{i}=a-s \leqq a,
$$


we have (for a $p$-soluble group $G$ )

$$
m_{p}(G) \leqq p\left(p^{a}-1\right) /(p-1) .
$$

Suppose now that $G$ is soluble. It is easily proved that

$$
p\left(p^{a}-1\right) /(p-1)+q\left(q^{b}-1\right) /(q-1)+\cdots<g
$$

unless $g$ is a prime power. Therefore, if $m(G)$ denotes the total number of maximal subgroups of $G$, we have

$$
m(G)=m_{p}(G)+m_{a}(G)+\cdots<g .
$$

It would be interesting to know whether (8.6) is valid for all finite groups. ( $(8.5)$ is certainly not universally true: the simple group of order 168 has 14 subgroups of index 7.)

It seems likely that every group with Eulerian polynomial of the form (8.2) is soluble, though I have not been able to prove this. The remarks which follow bear on this question.

$$
p|| G: G^{\prime} \mid \text { if., and only if, }(x-1) \mid \phi(G) .
$$

Proof. If $G$ has a normal subgroup of index $p$, then, by theorem 1 , $(x-1) \mid \phi(G)$. Conversely, suppose that $G$ has no normal subgroup of index $p$. Then the number of groups in each conjugacy class of subgroups of $p$-power index is divisible by $p$. Comparing coefficients of $y^{b} z^{c} \cdots$ in the sum formula

$$
f(G ; 1, y, z, \cdots)=\sum_{B \leqq G} \phi(H ; 1, y, z, \cdots),
$$

we see that the coefficient of $y^{b} z^{c} \cdot$ in $\phi(G ; 1, y, z, \cdots)$ is $\equiv 1(\bmod p)$ and so $(x-1) \nmid \phi(G)$.

(8.8) Corollary. If $\phi(G)$ has the form (8.2), $G^{\prime}<G$.

In fact, if $G$ is not .cyclic, $\phi_{1}(G)=0$. Hence one of $\rho(1), \sigma(1), \cdots$ is zero and so one of $(x-1),(y-1), \cdots$ divides $\phi(G)$. By $(8.7), G^{\prime}<G$.

(8.9) If $\phi(G)$ has the form (8.2) and $\mu_{G}(1) \neq 0, G$ is soluble.

In fact, since

$$
\mu_{G}(1)=\phi(G ; 0,0, \cdots)=\rho(0) \sigma(0) \cdots,
$$

the coefficients of $y^{b} z^{c} \cdots, x^{a} z^{c} \cdots$, in $\phi(G)$ are non-zero. Hence, by (3.2), $G$ has subgroups of each order $g / p^{a}, g / q^{b}, \cdots$ and so, by Hall's theorem, is soluble.

\section{References}

[1] Barnes, D. W., Lattice embeddings of prime power group, D. Phil. thesis, Oxford University (1959).

[2] Delsarte, P., Fonctions de Möbius sur les groupes abéliens finis, Ann. of Math. (2) 49 (1948), 600-609. 
[3] Gaschütz, W., Die Eulersche Funktion endlicher auflösbarer Gruppen, Illinois J. Maths. 3 (1959), 469-476.

[4] Hall, P., The Eulerian functions of a group, Quart. J. Maths. (Oxford Series) 7 (1936), $134-151$.

[5] Hall, P., A contribution to the theory of groups of prime-power order, Proc. London Math. Soc. (2) 36 (1933), 29-95.

[6] Kinosita, Y., On the enumeration of certain subgroups of a p-group, J. Osaka Inst. Sci. Tech. Part I I (1949), 13-20.

[7] Lazard, M., Sur les groupes nilpotents et les anneaux de Lie, Ann. Sc. de l'École Normale Supérieure 71 (1954), 101-190, (theorem 4.6, p. 176).

[8] Suzuki, M., Structure of a group and the structure of its lattice of subgroups, Springer (1956).

[9] Weisner, L., Abstract theory of inversion of finite series, Trans. Amer. Math. Soc. 38 (1935), 474-484.

[10] Yeh, Y., On prime power abelian groups, Bull. Amer. Math. Soc. 54 (1948), 323-327.

[11] Zassenhaus, H., The theory of groups, Chelsea (1949).

[12] Kemhadze, S. S., Soobščeniya Akad. Nauk Gruzin S.S.R. 11 (1950), 607-611.

\section{University of Sydney.}

